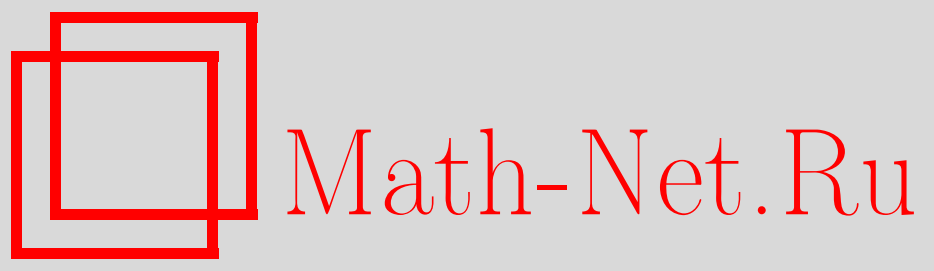

Е. K. Алексеев, Е. K. Kapeлина, Классификация корреляционно-иммунных и минимальных корреляционно-иммунных булевых функций от 4 и 5 переменных, Дискрет. матем., 2015, том 27, выпуск 1, 22-33

DOI: https://doi.org/10.4213/dm1312

Использование Общероссийского математического портала Math-Net.Ru подразумевает, что вы прочитали и согласны с пользовательским соглашением http://www . mathnet.ru/rus/agreement

Параметры загрузки:

IP : 3.81 .55 .215

26 апреля 2023 г., 13:43:22

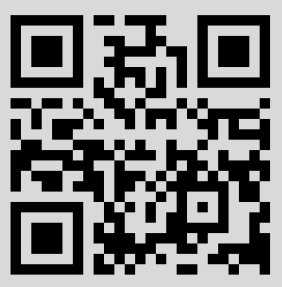




\title{
Классификация корреляционно-иммунных и минимальных корреляционно-иммунных булевых функций от 4 и 5 переменных
}

\author{
() 2015 г. Е. К. Алексеев*, Е. К. Карелина**
}

\begin{abstract}
Приведена классификация корреляционно-иммунных и минимальных корреляционно-иммунных булевых функций от 4 и 5 переменных относительно группы Джевонса. Приведены разложения представителей классов эквивалентности корреляционно-иммунных функций от 4 и 5 переменных на минимальные корреляционно-иммунные функции. Представлены характеристики различных разложений на минимальные корреляционно-иммунные функции функции-константы 1.

Работа выполнена при поддержке Российского фонда фундаментальных исследований, проект 12-01-00680-а.
\end{abstract}

Ключевые слова: криптография, корреляционно-иммунные функции, минимальные корреляционно-иммунные функции, классификация.

\section{1. Введение}

Корреляционная иммунность булевых функций означает отсутствие какой-либо статистической зависимости значения данной функции от значений некоторого подможества её аргументов или от значений определенных функций её аргументов. Корреляционно-иммунные функции позволяют противостоять методу восстановления ключа комбинирующего генератора, предложенному Зигенталером [6]. С геометрической точки зрения корреляционно-иммунная функция порядка $k$, зависящая от $n$ переменных, удалена от всех аффинных функций, отличных от констант и зависящих от $k$ и менее переменных, на расстояние $2^{n-1}$, то есть совпадает с ними ровно на половине входных наборов.

Существенный интерес представляет задача построения корреляционно-иммунных функций. Многие методы синтеза корреляционно-иммунных функций имеют итерационный характер - на каждом шаге новая функция строится с помощью функций, полученных на предыдущих шагах. При этом полученная таким образом функция зависит уже от большего числа переменных, чем её предшественницы. В таких методах существенное значение имеют «начальные точки»корреляционно-иммунные функции, с которых начинаются дальнейшие построения. В связи с этим возникает необходимость исследования функций от малого числа

\footnotetext{
* Место работы: МГУ им. М.В. Ломоносова, e-mail: geni-cmc@mail.ru

** Место работы: МГУ им. М.В. Ломоносова+, e-mail: karelinakaterina.cmc@gmail.com
} 
переменных, в частности, разработка их классификации относительно групп, сохраняющих определенный набор их свойств. Известны классификации различных множеств булевых функций относительно, например, полной аффинной группы и группы Джевонса (см., например, [8], [9], [10], [11], [12]). Отметим, что классификации булевых функций представляют существенный интерес не только для криптографии, но и для целого ряда научно-технических задач. Так, например, наличие классификаций облегчает задачу синтеза дискретных устройств, позволяет выбирать функции с наилучшими свойствами с точки зрения их применения в различных конкретных случаях.

Понятие минимальных корреляционно-иммунных функций было введено в [4]. Минимальная корреляционно-иммунная функция - это корреляционно-иммунная функция, из носителя которой нельзя исключить ни одного вектора так, чтобы полученная функция осталась корреляционно-иммунной. Сумма минимальных корреляционно-иммунных функций с не пересекающимися носителями является корреляционно-иммунной функцией (эта функция уже не является минимальной). Данное свойство дает основания полагать, что минимальные корреляционно-иммунные функции могут быть использованы при решении задач построения корреляционно-иммунных функций. Однако уровень изученности свойств минимальных функций на данный момент не позволяет строить какие-либо конкретные алгоритмы.

В данной работе содержатся некоторые результаты исследования свойств минимальных корреляционно-иммунных функций от 4 и 5 переменных. В работе впервые представлена классификация корреляционно-иммунных и минимальных корреляционно-иммунных булевых функций от 4 и 5 переменных относительно группы Джевонса. Приведены разложения представителей классов эквивалентности корреляционно-иммунных функций от 4 и 5 переменных на минимальные корреляционно-иммунные функции. Представлены характеристики различных разложений функции 1 на минимальные корреляционно-иммунные функции.

\section{2. Основные понятия и обозначения}

Пусть $\mathbb{F}_{2}-$ конечное поле из 2 элементов. Для произвольного $n \in \mathbb{N}$ определим $V_{n}=\left(\mathbb{F}_{2} \times \ldots \times \mathbb{F}_{2}\right)=\mathbb{F}_{2}^{n}$ - векторное пространство наборов длины $n$ с компонентами из поля $\mathbb{F}_{2}, V_{n}^{*}=V_{n} \backslash 0^{n}$, где $0^{n}=\underbrace{(0 \ldots 0)}_{n} \in V_{n}$.

Булевой функцией от $n$ переменных называется отображение из $V_{n}$ в $\mathbb{F}_{2}$. Множество всех булевых функций от $n$ переменных будем обозначать $\mathcal{F}_{n}$. Константные булевы функции обозначим через 1 и $\mathbf{0 .}$

Определение 1. Алгебраической степенью $\operatorname{deg}(f)$ булевой функции $f \in \mathcal{F}_{n}$ от $n$ переменных называется число переменных в самом длинном слагаемом АНФ (полиноме Жегалкина) функции $f$.

Обозначим множество $\mathcal{A}_{n}=\left\{f \in F_{n} \mid \operatorname{deg}(f) \leqslant 1\right\}$. Такие функции называются аффинными.

Определение 2. Носителем $1_{f}$ булевой функции $f \in \mathcal{F}_{n}$ называется множество $1_{f}=\left\{x \in V_{n} \mid f(x)=1\right\}$. Весом wt $(f)$ булевой функции $f \in \mathcal{F}_{n}$ называется мощность её носителя. 
Определение 3. Расстояние Хэмминга $\operatorname{dist}(f, g)$ между двумя булевыми функциями $f$ и $g$ равно мощности множества $\left\{x \in V_{n} \mid f(x) \neq g(x)\right\}$.

Определение 4. Преобразованием Уолша-Адамара булевой функции $f \in \mathcal{F}_{n}$ называется целочисленная функция на $V_{n}$, которая определяется равенством

$$
W_{f}(u)=\sum_{x \in V_{n}}(-1)^{f(x) \oplus<x, u>}
$$

(суммирование производится в действительной области). Для каждого $u \in V_{n}$ значение $W_{f}(u)$ называется коэффициентом Уолша-Адамара.

Определение 5. Нелинейностью $\mathrm{nl}(f)$ булевой функции $f \in \mathcal{F}_{n}$ называется расстояние Хэмминга до множества всех аффинных функций. То есть $\operatorname{nl}(f)=\operatorname{dist}\left(f, \mathcal{A}_{n}\right)$. Значение нелинейности можно вычислить по формуле:

$$
\operatorname{nl}(f)=2^{n-1}-\frac{1}{2} \max _{u \in V_{n}}\left|W_{f}(u)\right| .
$$

Определение 6. Булева функция $f \in \mathcal{F}_{n}$ называется корреляционно-иммунной порядка $m, 0<m \leqslant n$, если для любого вектора $u \in V_{n}$ такого, что $1 \leqslant \operatorname{wt}(u) \leqslant m$, выполнено равенство $W_{f}(u)=0$.

Корреляционно-иммунная функция порядка $m$ является корреляционно-иммунной любого меньшего порядка, поэтому введем обозначение

$$
\operatorname{cor}(f)=\max \{m \in \mathbb{N} \mid f-\text { корреляционно-иммунная порядка } m\} \text {. }
$$

Для булевой функции $f \in \mathcal{F}_{n}$ и произвольного вектора $u \in V_{n}$ будем обозначать через $f^{u}$ функцию $f^{u}: x \rightarrow f(x \oplus u)$.

Определение 7. Производной функции $f \in \mathcal{F}_{n}$ по направлению $u \in V_{n}^{*}$ называется функция $D_{u} f=f^{u} \oplus f$.

Определение 8. Пространством линейных структур функции $f \in \mathcal{F}_{n}$ называется множество $L_{f}=\left\{u \in V_{n} \mid D_{u} f=\right.$ const $\}$. Все $u$, входящие в это множество, называются нетривиальными линейными структурами функции $f$. Множество булевых функций $f \in \mathcal{F}_{n}$, имеющих нетривиальные линейные структуры, обозначается через $\mathcal{F}_{n}^{0}$.

В работе [5] была введена следующая мера нелинейности для функции $f \in \mathcal{F}_{n}$ :

$$
\delta(f)=\operatorname{dist}\left(f, \mathcal{F}_{n}^{0}\right) .
$$

Пусть $A-(n \times k)$-матрица над $\mathbb{F}_{2}$, а $f-$ булева функция от $k$ переменных. Через $f^{A}$ будем обозначать функцию от $n$ переменных, определенную следующим образом: $f^{A}: x \rightarrow f(x A)$.

Определение 9. Порядком алгебраической вырожденности $A D(f)$ булевой функции $f \in \mathcal{F}_{n}$ называется максимально возможное значение $n-k$, где целое число $0 \leqslant k \leqslant n$ таково, что существуют такие функция $g \in \mathcal{F}_{k}$ и $(n \times k)$-матрица $D$ над $\mathbb{F}_{2}$, что выполнено равенство $f=g^{D}$.

Определение 10. Функции, для которых $A D(f)>0$, называются алгебраически вырожденными. Множество всех алгебраически вырожденных функций от $n$ переменных обозначим через $D G(n)=\left\{f \in \mathcal{F}_{n} \mid A D(f)>0\right\}$. 
Определение 11. Невырожденностью функции $f \in \mathcal{F}_{n}$ называется значение

$$
\rho(f)=\operatorname{dist}(f, D G(n)) .
$$

Определение 12. Группой Джевонса $\mathcal{D}_{n}$ называется группа, состоящая из $2^{n} \cdot n$ ! подстановок вида

$$
g:\left(x_{1}, \ldots, x_{n}\right) \mapsto\left(x_{j_{1}} \oplus a_{1}, \ldots, x_{j_{n}} \oplus a_{n}\right),
$$

где $\left(\begin{array}{ccc}1 & \ldots & n \\ j_{1} & \ldots & j_{n}\end{array}\right)$ - подстановка на множестве $\{1, \ldots, n\}$ и

$$
a=\left(a_{1}, \ldots, a_{n}\right) \in V_{n}
$$

Обозначим множество всех корреляционно-иммунных булевых функций как минимум первого порядка, зависящих от $n$ переменных, через $C I(n)$.

В работе [4] было введено понятие минимальной корреляционно-иммунной функции.

Определение 13. Функция $f \in C I(n)$ называется минимальной корреляционно-иммунной функцией, если не существует такой функции $g \in C I(n)$, что $f \cdot g=g$.

Следующие свойства минимальных корреляционно-иммунных функций доказаны в [4].

Теорема 1 ([4]). Функция $f \in C I(n)$ является минимальной корреляционно-иммунной функцией тогда и только тогда, когда $f$ невозможно разложить в сумму двух корреляционно-иммунных функиий с не пересекающимися носителями (т.е. $f$ нельзя представить в виде суммы $f=g \oplus h$, где $g, h \in C I(n) u h \cdot g=0)$.

Теорема 2 ([4]). Для любой минимальной корреляционно-иммунной булевой функции $f$ выполнено неравенство $\operatorname{wt}(f)<2^{n-1}$, если $n \geqslant 4$.

Теорема 3 ([4]). Для любой минимальной коррелячионно-иммунной булевой функиии $f$ справедливо неравенство $\operatorname{cor}(f) \leqslant 2$.

Теорема 4 ([4]). Любая функиия $f \in C I(n)$ может быть разложена в сумму минимальных коррелячионно-иммунных булевых функиий с попарно не пересекающимися носителями.

Таким образом, зная все минимальные корреляционно-иммунные функции от $n$ переменных, можно получить все функции из $C I(n)$, рассматривая всевозможные суммы минимальных корреляционно-иммунных функций с попарно не пересекающимися носителями.

Декомпозиция константы $\mathbf{1}$ на минимальные корреляционно-иммунные функции позволяет построить множества $L_{1}, \ldots, L_{T}$, являющиеся линейными подпространствами пространства $\mathcal{F}_{n}$ и состоящие из корреляционно-иммунных функций, объединение которых дает все множество $C I(n)$.

Множество всех минимальных корреляционно-иммунных функций от $n$ переменных обозначается через $M C I(n)$.

Пусть $G$ - группа, состоящая из преобразований множества $V_{n}$, и $g \in G$. Через $f^{g}$ обозначим функцию $f^{g}(x)=f(g(x))$. Через $f^{G}$ обозначим множество $f^{G}=\left\{f^{g} \mid g \in G\right\}$. 
Введем отношение на множестве функций:

$$
f \sim h \Leftrightarrow \exists g \in G: f^{g}=h .
$$

Это отношение - отношение эквивалентности. Множество $f^{G}-$ класс эквивалентности, представителем которого является функция $f$.

Под классификацией некоторого множества $M$ булевых функций относительно некоторой группы $G$ понимается множество всех различных классов эквивалентности $f^{G}$, где $f \in M$. Класс эквивалентности далее будет задаваться любым его представителем.

Для элементов группы $\mathcal{D}_{n}$ справедливо следующее утверждение.

Теорема 5 ([1]). Для любой корреляционно-иммунной (минимальной корреляицонно-иммунной) порядка $m, 0<m \leqslant n$, функиии $f \in \mathcal{F}_{n}$ и элемента $g \in$ $\mathcal{D}_{n}$ функиия $f^{g} \in \mathcal{F}_{n}$ также является корреляционно-иммунной (минимальной корреляционно-иммунной) функцией порядка $m$.

Обозначим через $\widehat{C I}(n)$ множество классов эквивалентности. Для удобства представления обозначим через $\widehat{C I_{w}}(n)$ и $\widehat{C I^{c}}(n)$ множество классов эквивалентности, в котором любая функция имеет вес $w$, и множество классов эквивалентности, в котором любая функция имеет порядок корреляционной иммунности, равный $c$, соответственно. Через $\widehat{C I_{w}^{c}}(n)$ обозначим множество классов эквивалентности, в котором любая функция имеет вес $w$ и порядок корреляционной иммунности, равный $c$.

Обозначим через $C I_{w}(n)$ и $C I^{c}(n)$ множество корреляционно-иммунных функций, в котором любая функция имеет вес $w$, и множество корреляционно-иммунных функций, в котором любая функция имеет порядок корреляционной иммунности, равный $c$, соответственно. Через $C I_{w}^{c}(n)$ обозначим множество корреляционно-иммунных функций, в котором любая функция имеет вес $w$ и порядок корреляционной иммунности, равный $c$.

Аналогичным образом вводятся обозначения для множества $M C I(n)$.

Везде ниже для описания булевой функции приводится ее таблица истинности (векторы расположены в лексикографическом порядке слева направо) в сокращенной форме - четверки подряд стоящих двоичных значений представлены в виде числа в шестнадцатеричной системе счисления. Так, например, таблица истинности булевой функции $f\left(x_{1}, x_{2}, x_{3}\right)=0 \mathrm{x} 8 \mathrm{C}$ от 3 переменных такова:

$$
\begin{array}{cc}
x_{1} & 00001111 \\
x_{2} & 00110011 \\
x_{3} & 01010101 \\
\hline f & \underbrace{1000}_{8} \underbrace{1100}_{\mathrm{C}}
\end{array}
$$

\section{3. Классификация корреляционно-иммунных буле- вых функций}

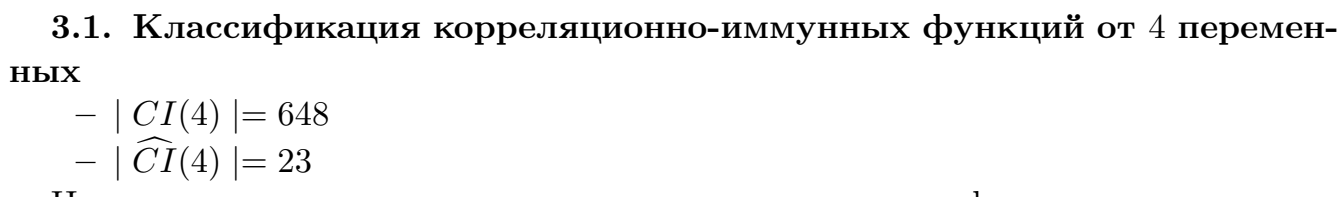

Ниже представлены некоторые характеристики классификации корреляционно-иммунных функций от 4 переменных относительно группы Джевонса. 


\begin{tabular}{|c|c|c||c|c|c|c|c|}
\hline$w$ & $\left|\widehat{C I_{w}}(4)\right|$ & $\left|C I_{w}(4)\right|$ & $c$ & $\left|\widehat{C I^{c}}(4)\right|$ & $\left|C I^{c}(4)\right|$ & $\left|\widehat{C I_{8}^{c}}(4)\right|$ & $\left|C I_{8}^{c}(4)\right|$ \\
\hline 0 & 1 & 1 & 1 & 19 & 636 & 5 & 212 \\
\hline 2 & 1 & 8 & 2 & 1 & 8 & 1 & 8 \\
\hline 4 & 3 & 52 & 3 & 1 & 2 & 1 & 2 \\
\hline 6 & 3 & 152 & 4 & 2 & 2 & 0 & 0 \\
\hline 8 & 7 & 222 & & \multicolumn{3}{|c|}{} \\
\hline
\end{tabular}

В следующей таблице приведена полная классификация корреляционно-иммунных функций от 4 переменных.

\begin{tabular}{|c|c|c|c|c|c|c|c|}
\hline$f$ & $\mathrm{wt}(f)$ & $\operatorname{deg}(f)$ & $\mathrm{nl}(f)$ & $\operatorname{cor}(f)$ & $\rho(f)$ & $\delta(f)$ & $\mid f^{\mathcal{D}_{n}}$ \\
\hline 0x0000 & 0 & 0 & 0 & 4 & 0 & 0 & 1 \\
\hline 0x0180 & 2 & 3 & 2 & 1 & 0 & 0 & 8 \\
\hline 0x03c0 & 4 & 2 & 4 & 1 & 0 & 0 & 16 \\
\hline 0x0660 & 4 & 2 & 4 & 1 & 0 & 0 & 12 \\
\hline 0x0690 & 4 & 2 & 4 & 1 & 0 & 0 & 24 \\
\hline 0x07e0 & 6 & 3 & 2 & 1 & 0 & 0 & 48 \\
\hline 0x1668 & 6 & 3 & 2 & 1 & 0 & 0 & 8 \\
\hline 0x1698 & 6 & 3 & 4 & 1 & 2 & 2 & 96 \\
\hline 0x0ff0 & 8 & 1 & 0 & 1 & 0 & 0 & 12 \\
\hline 0x17e8 & 8 & 2 & 4 & 1 & 0 & 0 & 32 \\
\hline 0x1bd8 & 8 & 2 & 4 & 1 & 0 & 0 & 24 \\
\hline 0x1be4 & 8 & 2 & 4 & 1 & 0 & 0 & 96 \\
\hline 0x1ee1 & 8 & 2 & 4 & 1 & 0 & 0 & 48 \\
\hline 0x3cc3 & 8 & 1 & 0 & 2 & 0 & 0 & 8 \\
\hline 0x6996 & 8 & 1 & 0 & 3 & 0 & 0 & 2 \\
\hline 0x1ff8 & 10 & 3 & 2 & 1 & 0 & 0 & 48 \\
\hline 0x3dda & 10 & 3 & 4 & 1 & 2 & 2 & 96 \\
\hline 0x6bd6 & 10 & 3 & 2 & 1 & 0 & 0 & 8 \\
\hline 0x6ff6 & 12 & 2 & 4 & 1 & 0 & 0 & 12 \\
\hline 0x3ffc & 12 & 2 & 4 & 1 & 0 & 0 & 16 \\
\hline 0x6ff9 & 12 & 2 & 4 & 1 & 0 & 0 & 24 \\
\hline 0x7ffe & 14 & 3 & 2 & 1 & 0 & 0 & 8 \\
\hline 0xffff & 16 & 0 & 0 & 4 & 0 & 0 & 1 \\
\hline
\end{tabular}

3.2. Классификация корреляционно-иммунных функций от 5 переменных

$$
\begin{aligned}
& -|C I(5)|=3140062 \\
& -|\widehat{C I}(5)|=1524
\end{aligned}
$$

Ниже представлены некоторые характеристики классификации корреляционно-иммунных функций от 5 переменных относительно группы Джевонса. 


\begin{tabular}{|c|c|c|c|c|c|c|c|}
\hline$w$ & $\left|\widehat{C I_{w}}(5)\right|$ & $C I_{w}(5)$ & $c$ & $\widehat{C I^{c}}(5)$ & $C I^{c}(5)$ & $\overline{C I_{16}^{c}}(5)$ & $C I_{16}^{c}(5)$ \\
\hline 0 & 1 & 1 & 1 & 1511 & 3139004 & 359 & 807428 \\
\hline 2 & 1 & 16 & 2 & 9 & 1044 & 5 & 540 \\
\hline 4 & 4 & 320 & 3 & 1 & 10 & 1 & 10 \\
\hline 6 & 8 & 3824 & 4 & 1 & 2 & 1 & 2 \\
\hline 8 & 36 & 27640 & 5 & 2 & 2 & 0 & 0 \\
\hline 10 & 73 & 123600 & & & & & \\
\hline 12 & 190 & 353120 & & & & & \\
\hline 14 & 266 & 657520 & & & & & \\
\hline 16 & 366 & 807980 & & & & & \\
\hline
\end{tabular}

Из таблиц видно, что количество классов эквивалентности весьма велико, поэтому приведем примеры представителей лишь некоторых из них. Рассмотрим те представители классов эквивалентности, у которых порядок корреляционной иммунности равен 2,3 и 4 .

\begin{tabular}{|c|c|c|c|c|c|c|c|}
\hline$f$ & $\operatorname{wt}(f)$ & $\operatorname{deg}(f)$ & $\operatorname{nl}(f)$ & $\operatorname{cor}(f)$ & $\rho(f)$ & $\delta(f)$ & $\mid f^{\mathcal{D}_{n}}$ \\
\hline 0x06609009 & 8 & 2 & 8 & 2 & 0 & 0 & 60 \\
\hline 0x1698a443 & 12 & 3 & 12 & 2 & 6 & 6 & 192 \\
\hline 0x0ff0f00f & 16 & 1 & 0 & 2 & 0 & 0 & 20 \\
\hline 0x17e8e817 & 16 & 2 & 8 & 2 & 0 & 0 & 80 \\
\hline 0x1bd8e427 & 16 & 2 & 8 & 2 & 0 & 0 & 120 \\
\hline 0x1be4e41b & 16 & 2 & 8 & 2 & 0 & 0 & 240 \\
\hline 0x1ee1e11e & 16 & 2 & 8 & 2 & 0 & 0 & 80 \\
\hline 0x3cc3c33c & 16 & 1 & 0 & 3 & 0 & 0 & 10 \\
\hline 0x69969669 & 16 & 1 & 0 & 4 & 0 & 0 & 2 \\
\hline 0x3ddae697 & 20 & 3 & 12 & 2 & 6 & 6 & 192 \\
\hline 0x6ff6f99f & 24 & 2 & 8 & 2 & 0 & 0 & 60 \\
\hline
\end{tabular}

3.3. Классификация минимальных корреляционно-иммунных функций от 4 переменных

$$
\begin{aligned}
& -|M C I(4)|=32 \\
& -|\widehat{M C I}(4)|=2
\end{aligned}
$$

Далее представлены все минимальные корреляционно-иммунные функции от 4 переменных: 


\begin{tabular}{|c|c|c|c|c|c|c|}
\hline$f$ & $\mathrm{wt}(f)$ & $\mathrm{deg}(f)$ & $\mathrm{nl}(f)$ & $\operatorname{cor}(f)$ & $\rho(f)$ & $\delta(f)$ \\
\hline 0x0180 & 2 & 3 & 2 & 1 & 0 & 0 \\
\hline 0x0240 & 2 & 3 & 2 & 1 & 0 & 0 \\
\hline 0x0420 & 2 & 3 & 2 & 1 & 0 & 0 \\
\hline 0x0810 & 2 & 3 & 2 & 1 & 0 & 0 \\
\hline 0x1008 & 2 & 3 & 2 & 1 & 0 & 0 \\
\hline 0x2004 & 2 & 3 & 2 & 1 & 0 & 0 \\
\hline 0x4002 & 2 & 3 & 2 & 1 & 0 & 0 \\
\hline 0x8001 & 2 & 3 & 2 & 1 & 0 & 0 \\
\hline 0x0690 & 4 & 2 & 4 & 1 & 0 & 0 \\
\hline 0x0960 & 4 & 2 & 4 & 1 & 0 & 0 \\
\hline 0x1284 & 4 & 2 & 4 & 1 & 0 & 0 \\
\hline 0x1482 & 4 & 2 & 4 & 1 & 0 & 0 \\
\hline 0x1824 & 4 & 2 & 4 & 1 & 0 & 0 \\
\hline 0x1842 & 4 & 2 & 4 & 1 & 0 & 0 \\
\hline 0x1881 & 4 & 2 & 4 & 1 & 0 & 0 \\
\hline 0x2148 & 4 & 2 & 4 & 1 & 0 & 0 \\
\hline 0x2418 & 4 & 2 & 4 & 1 & 0 & 0 \\
\hline 0x2442 & 4 & 2 & 4 & 1 & 0 & 0 \\
\hline 0x2481 & 4 & 2 & 4 & 1 & 0 & 0 \\
\hline 0x2841 & 4 & 2 & 4 & 1 & 0 & 0 \\
\hline 0x4128 & 4 & 2 & 4 & 1 & 0 & 0 \\
\hline 0x4218 & 4 & 2 & 4 & 1 & 0 & 0 \\
\hline 0x4224 & 4 & 2 & 4 & 1 & 0 & 0 \\
\hline 0x4281 & 4 & 2 & 4 & 1 & 0 & 0 \\
\hline 0x4821 & 4 & 2 & 4 & 1 & 0 & 0 \\
\hline 0x6009 & 4 & 2 & 4 & 1 & 0 & 0 \\
\hline 0x8118 & 4 & 2 & 4 & 1 & 0 & 0 \\
\hline 0x8124 & 4 & 2 & 4 & 1 & 0 & 0 \\
\hline 0x8142 & 4 & 2 & 4 & 1 & 0 & 0 \\
\hline 0x8214 & 4 & 2 & 4 & 1 & 0 & 0 \\
\hline 0x8412 & 4 & 2 & 4 & 1 & 0 & 0 \\
\hline 0x9006 & 4 & 2 & 4 & 1 & 0 & 0 \\
\hline
\end{tabular}

\begin{tabular}{|c|c|c|}
\hline$w$ & $\left|\widehat{M C I}_{w}(4)\right|$ & $\left|M C I_{w}(4)\right|$ \\
\hline 2 & 1 & 8 \\
\hline 4 & 1 & 24 \\
\hline
\end{tabular}

Классификация минимальных корреляционно-иммунных функций от 4 переменных:

\begin{tabular}{|c|c|c|c|c|c|c|c|}
\hline$f$ & $\mathrm{wt}(f)$ & $\operatorname{deg}(f)$ & $\mathrm{nl}(f)$ & $\operatorname{cor}(f)$ & $\rho(f)$ & $\delta(f)$ & $\left|f^{\mathcal{D}_{n}}\right|$ \\
\hline 0x0180 & 2 & 3 & 2 & 1 & 0 & 0 & 8 \\
\hline 0x0690 & 4 & 2 & 4 & 1 & 0 & 0 & 24 \\
\hline
\end{tabular}

3.4. Классификация минимальных корреляционно-иммунных функций от 5 переменных

$-|M C I(5)|=1240$

$-|\widehat{M C I}(5)|=6$ 


\begin{tabular}{|c|c|c|}
\hline$w$ & $\left|\widehat{M C I}_{w}(5)\right|$ & $\left|M C I_{w}(5)\right|$ \\
\hline 2 & 1 & 16 \\
\hline 4 & 2 & 200 \\
\hline 6 & 2 & 864 \\
\hline 8 & 1 & 160 \\
\hline
\end{tabular}

Классификация минимальных корреляционно-иммунных функций от 5 переменных:

\begin{tabular}{|c|c|c|c|c|c|c|c|}
\hline$f$ & $\mathrm{wt}(f)$ & $\operatorname{deg}(f)$ & $\mathrm{nl}(f)$ & $\operatorname{cor}(f)$ & $\rho(f)$ & $\delta(f)$ & $\left|f^{\mathcal{D}_{n}}\right|$ \\
\hline 0x00018000 & 2 & 4 & 2 & 1 & 0 & 0 & 16 \\
\hline 0x00069000 & 4 & 3 & 4 & 1 & 0 & 0 & 80 \\
\hline 0x00182400 & 4 & 3 & 4 & 1 & 0 & 0 & 120 \\
\hline 0x01186008 & 6 & 4 & 6 & 1 & 4 & 4 & 480 \\
\hline 0x01188240 & 6 & 4 & 6 & 1 & 4 & 4 & 384 \\
\hline 0x01686801 & 8 & 3 & 8 & 1 & 0 & 0 & 160 \\
\hline
\end{tabular}

\section{4. Разложения корреляционно-иммунных фун- кций от 4 и 5 переменных на минимальные корреляционно-иммунные функции}

Разложить данную корреляционно-иммунную функцию $f \in \mathcal{F}_{n}$ в сумму минимальных корреляционно-иммунных функций с не пересекающимися носителями - значит представить $f$ в виде $f=m_{1} \oplus \ldots \oplus m_{t}$, где $m_{i} \in M C I(n)$ для любого $i$ и $m_{i} \cdot m_{j}=\mathbf{0}$ при $i \neq j$. В общем случае разложить функцию $f \in C I(n)$ на минимальные можно многими способами, то есть разложение не единственно. Весовой сигнатурой $\left(w_{1}^{t_{1}}, w_{2}^{t_{2}}, \ldots, w_{s}^{t_{s}}\right)$ разложения некоторой функции $f$ (далее просто сигнатурой) будем называть множество разложений функции $f$ вида

$$
f=m_{1,1} \oplus m_{1,2} \oplus \ldots \oplus m_{1, t_{1}} \oplus \ldots \oplus m_{s, 1} \oplus m_{s, 2} \oplus \ldots \oplus m_{s, t_{s}}
$$

где $m_{i, j} \in M C I(n), \operatorname{wt}\left(m_{i, j}\right)=w_{i}$ и $\sum_{i} t_{i} \cdot w_{i}=\operatorname{wt}(f)$ (в разложении все минимальные корреляционно-иммунные функции сгруппированы по весам). В общем случае для данной функции $f \in C I(n)$ может существовать множество различных сигнатур.

Заметим, что любой минимальной корреляционно-иммунной булевой функции $f$ соответствует одна сигнатура $\left(\mathrm{wt}(f)^{1}\right)$ мощности 1.

4.1. Разложения корреляционно-иммунных функций от 4 переменных на минимальные корреляционно-иммунные функции. В этом разделе приведены сигнатуры представителей всех классов эквивалентности множества $C I(4)$. Приведены также мощности каждой из сигнатур (то есть количества различных разложений с данной сигнатурой). 


\begin{tabular}{|c|l|l|}
\hline$f$ & Сигнатура & $\begin{array}{l}\text { Мощность } \\
\text { сигнату- } \\
\text { ры }\end{array}$ \\
\hline 0x0180 & $\left(2^{1}\right)$ & 1 \\
\hline 0x03c0 & $\left(2^{2}\right)$ & 1 \\
\hline 0x0660 & $\left(2^{2}\right)$ & 1 \\
\hline 0x0690 & $\left(4^{1}\right)$ & 1 \\
\hline 0x07e0 & $\left(2^{3}\right)$ & 1 \\
\hline 0x0ff0 & $\left(2^{4}\right)$ & 1 \\
& $\left(4^{2}\right)$ & 1 \\
\hline 0x1668 & $\left(2^{3}\right)$ & 1 \\
\hline 0x1698 & $\left(2^{1}, 4^{1}\right)$ & 1 \\
\hline 0x17e8 & $\left(2^{4}\right)$ & 1 \\
\hline 0x1bd8 & $\left(2^{4}\right)$ & 1 \\
\hline 0x1be4 & $\left(2^{2}, 4^{1}\right)$ & 1 \\
& $\left(4^{2}\right)$ & 1 \\
\hline 0x1ee1 & $\left(2^{2}, 4^{1}\right)$ & 1 \\
\hline 0x1ff8 & $\left(2^{1}, 4^{2}\right)$ & 1 \\
& $\left(2^{5}\right)$ & 1 \\
\hline
\end{tabular}

\begin{tabular}{|c|l|l|}
\hline$f$ & Сигнатура & $\begin{array}{l}\text { Мощность } \\
\text { сигнату- } \\
\text { ры }\end{array}$ \\
\hline 0x3cc3 & $\left(4^{2}\right)$ & 3 \\
\hline 0x3dda & $\left(2^{1}, 4^{2}\right)$ & 2 \\
& $\left(2^{3}, 4^{1}\right)$ & 1 \\
\hline 0x3ffc & $\left(2^{2}, 4^{2}\right)$ & 3 \\
& $\left(2^{6}\right)$ & 1 \\
& $\left(4^{3}\right)$ & 2 \\
\hline 0x6996 & $\left(2^{4}\right)$ & 1 \\
\hline 0x6bd6 & $\left(2^{5}\right)$ & 1 \\
\hline 0x6ff6 & $\left(2^{2}, 4^{2}\right)$ & 2 \\
& $\left(2^{6}\right)$ & 1 \\
\hline 0x6ff9 & $\left(2^{2}, 4^{2}\right)$ & 4 \\
& $\left(2^{4}, 4^{1}\right)$ & 1 \\
& $\left(4^{3}\right)$ & 5 \\
\hline 0x7ffe & $\left(2^{1}, 4^{3}\right)$ & 8 \\
& $\left(2^{3}, 4^{2}\right)$ & 6 \\
& $\left(2^{7}\right)$ & 1 \\
\hline
\end{tabular}

4.2. Разложения корреляционно-иммунных функций от 5 переменных на минимальные корреляционно-иммунные функции В этом разделе приведены сигнатуры (и их мощности) тех представителей классов эквивалентности множества $C I(5)$, которые имеют порядок корреляционной иммунности, равный 2 , 3 и 4.

\begin{tabular}{|c|l|l|}
\hline$f$ & Сигнатура & $\begin{array}{l}\text { Мощность } \\
\text { сигнату- } \\
\text { ры }\end{array}$ \\
\hline 0x06609009 & $\left(4^{2}\right)$ & 2 \\
\hline 0x0ff0f00f & $\left(4^{4}\right)$ & 81 \\
& $\left(8^{2}\right)$ & 4 \\
\hline 0x1698a443 & $\left(2^{1}, 4^{1}, 6^{1}\right)$ & 5 \\
& $\left(6^{2}\right)$ & 1 \\
\hline 0x17e8e817 & $\left(4^{1}, 6^{2}\right)$ & 72 \\
& $\left(4^{4}\right)$ & 53 \\
& $\left(8^{2}\right)$ & 1 \\
\hline 0x1bd8e427 & $\left(4^{1}, 6^{2}\right)$ & 96 \\
& $\left(4^{4}\right)$ & 34 \\
\hline 0x1be4e41b & $\left(2^{1}, 4^{2}, 6^{1}\right)$ & 8 \\
& $\left(2^{2}, 4^{3}\right)$ & 4 \\
& $\left(2^{2}, 6^{2}\right)$ & 4 \\
& $\left(2^{4}, 4^{2}\right)$ & 3 \\
& $\left(4^{4}\right)$ & 4 \\
\hline 0x1ee1e11e & $\left(2^{2}, 6^{2}\right)$ & 12 \\
& $\left(2^{4}, 4^{2}\right)$ & 3 \\
& $\left(8^{2}\right)$ & 3 \\
\hline 0x3ddae697 & $\left(2^{1}, 4^{3}, 6^{1}\right)$ & 170 \\
\hline
\end{tabular}

\begin{tabular}{|c|l|l|}
\hline$f$ & Сигнатура & $\begin{array}{l}\text { Мощность } \\
\text { сигнату- } \\
\text { ры }\end{array}$ \\
\hline 0x6ff6f99f & $\left(2^{1}, 4^{1}, 6^{3}\right)$ & 1184 \\
$\left(2^{1}, 4^{2}, 6^{1}, 8^{1}\right)$ & 160 \\
$\left(2^{1}, 4^{4}, 6^{1}\right)$ & 2920 \\
& $\left(2^{1}, 6^{1}, 8^{2}\right)$ & 8 \\
& $\left(2^{2}, 4^{2}, 6^{2}\right)$ & 2956 \\
& $\left(2^{2}, 4^{3}, 8^{1}\right)$ & 96 \\
& $\left(2^{2}, 4^{5}\right)$ & 784 \\
& $\left(2^{3}, 4^{1}, 6^{1}, 8^{1}\right)$ & 32 \\
& $\left(2^{3}, 4^{3}, 6^{1}\right)$ & 1216 \\
$\left(2^{3}, 6^{3}\right)$ & 288 \\
& $\left(2^{4}, 4^{1}, 6^{2}\right)$ & 368 \\
$\left(2^{4}, 4^{4}\right)$ & 374 \\
$\left(2^{5}, 4^{2}, 6^{1}\right)$ & 264 \\
$\left(2^{6}, 4^{1}, 8^{1}\right)$ & 8 \\
$\left(2^{6}, 4^{3}\right)$ & 32 \\
$\left(2^{6}, 6^{2}\right)$ & 36 \\
$\left(2^{8}, 4^{2}\right)$ & 2 \\
$\left(4^{1}, 6^{2}, 8^{1}\right)$ & 240 \\
$\left(4^{2}, 8^{2}\right)$ & 16 \\
& &
\end{tabular}




\begin{tabular}{|l|l|l|}
$\left(2^{1}, 6^{3}\right)$ & 20 \\
$\left(2^{2}, 4^{1}, 6^{2}\right)$ & 100 \\
$\left(2^{2}, 4^{4}\right)$ & 50 \\
$\left(2^{3}, 4^{2}, 6^{1}\right)$ & 90 \\
$\left(2^{4}, 4^{3}\right)$ & 5 \\
$\left(2^{4}, 6^{2}\right)$ & 5 \\
$\left(2^{5}, 4^{1}, 6^{1}\right)$ & 5 \\
$\left(4^{2}, 6^{2}\right)$ & 70 \\
$\left(4^{5}\right)$ & 4 \\
\hline
\end{tabular}

\begin{tabular}{|l|l|l|} 
& $\left(4^{3}, 6^{2}\right)$ & 2552 \\
& $\left(4^{6}\right)$ & 880 \\
& $\left(6^{4}\right)$ & 112 \\
\hline 0x3cc3c33c & $\left(2^{2}, 4^{3}\right)$ & 8 \\
& $\left(2^{4}, 4^{2}\right)$ & 6 \\
& $\left(2^{8}\right)$ & 1 \\
& $\left(4^{4}\right)$ & 9 \\
\hline $0 \times 69969669$ & $\left(4^{4}\right)$ & 110 \\
\hline
\end{tabular}

4.3. Разложение функции-константы 1 на минимальные корреляционно-иммунные функции Рассмотрим отдельно разложения функции-константы 1 на минимальные корреляционно-иммунные функции.

Для функции $\mathbf{1} \in \mathcal{F}_{4}$ существует всего 75 разложений. Для функции $\mathbf{1} \in \mathcal{F}_{5}-$ уже 40214 156. Далее для этих функций приведены сигнатуры и их мощности.

\begin{tabular}{|c|l|l|}
\hline$f$ & Сигнатура & $\begin{array}{l}\text { Мощность } \\
\text { сигнату- } \\
\text { ры }\end{array}$ \\
\hline 0xffff & $\left(2^{2}, 4^{3}\right)$ & 32 \\
& $\left(2^{4}, 4^{2}\right)$ & 12 \\
& $\left(2^{8}\right)$ & 1 \\
& $\left(4^{4}\right)$ & 30 \\
\hline 0xfffffff & $\left(2^{1}, 4^{1}, 6^{3}, 8^{1}\right)$ & 355200 \\
& $\left(2^{1}, 4^{2}, 6^{1}, 8^{2}\right)$ & 33600 \\
& $\left(2^{1}, 4^{3}, 6^{3}\right)$ & 7948800 \\
& $\left(2^{1}, 4^{4}, 6^{1}, 8^{1}\right)$ & 456960 \\
& $\left(2^{1}, 4^{6}, 6^{1}\right)$ & 3302880 \\
& $\left(2^{1}, 6^{5}\right)$ & 517632 \\
& $\left(2^{10}, 4^{3}\right)$ & 720 \\
& $\left(2^{10}, 6^{2}\right)$ & 432 \\
& $\left(2^{12}, 4^{2}\right)$ & 100 \\
& $\left(2^{2}, 4^{1}, 6^{4}\right)$ & 2077440 \\
& $\left(2^{2}, 4^{2}, 6^{2}, 8^{1}\right)$ & 504960 \\
& $\left(2^{2}, 4^{3}, 8^{2}\right)$ & 4960 \\
& $\left(2^{2}, 4^{4}, 6^{2}\right)$ & 6860880 \\
& $\left(2^{2}, 4^{5}, 8^{1}\right)$ & 114240 \\
& $\left(2^{2}, 4^{7}\right)$ & 596400 \\
& $\left(2^{2}, 6^{2}, 8^{2}\right)$ & 12000 \\
& $\left(2^{3}, 4^{1}, 6^{1}, 8^{2}\right)$ & 3840 \\
& $\left(2^{3}, 4^{2}, 6^{3}\right)$ & 2896000 \\
& $\left(2^{3}, 4^{3}, 6^{1}, 8^{1}\right)$ & 252480 \\
& $\left(2^{3}, 4^{5}, 6^{1}\right)$ & 2192640 \\
& $\left(2^{3}, 6^{3}, 8^{1}\right)$ & 42240 \\
& $\left(2^{4}, 4^{1}, 6^{2}, 8^{1}\right)$ & 72960 \\
& $\left(2^{4}, 4^{2}, 8^{2}\right)$ & 2160 \\
$\left(2^{4}, 4^{3}, 6^{2}\right)$ & 1808160 \\
\hline
\end{tabular}

\begin{tabular}{|c|c|c|}
\hline$f$ & Сигнатура & $\begin{array}{l}\text { Мощность } \\
\text { сигнату- } \\
\text { ры }\end{array}$ \\
\hline 0xffffffff & $\begin{array}{l}\left(2^{4}, 4^{4}, 8^{1}\right) \\
\left(2^{4}, 4^{6}\right) \\
\left(2^{4}, 6^{4}\right) \\
\left(2^{5}, 4^{1}, 6^{3}\right) \\
\left(2^{5}, 4^{2}, 6^{1}, 8^{1}\right) \\
\left(2^{5}, 4^{4}, 6^{1}\right) \\
\left(2^{5}, 6^{1}, 8^{2}\right) \\
\left(2^{6}, 4^{2}, 6^{2}\right) \\
\left(2^{6}, 4^{3}, 8^{1}\right) \\
\left(2^{6}, 4^{5}\right) \\
\left(2^{7}, 4^{1}, 6^{1}, 8^{1}\right) \\
\left(2^{7}, 4^{3}, 6^{1}\right) \\
\left(2^{7}, 6^{3}\right) \\
\left(2^{8}, 4^{1}, 6^{2}\right) \\
\left(2^{8}, 4^{4}\right) \\
\left(2^{8}, 8^{2}\right) \\
\left(2^{9}, 4^{2}, 6^{1}\right) \\
\left(2^{16}\right) \\
\left(4^{1}, 6^{2}, 8^{2}\right) \\
\left(4^{2}, 6^{4}\right) \\
\left(4^{3}, 6^{2}, 8^{1}\right) \\
\left(4^{4}, 8^{2}\right) \\
\left(4^{5}, 6^{2}\right) \\
\left(4^{6}, 8^{1}\right) \\
\left(4^{8}\right) \\
\left(6^{4}, 8^{1}\right) \\
\left(8^{4}\right) \\
\end{array}$ & $\begin{array}{l}44160 \\
264340 \\
145080 \\
238080 \\
28800 \\
506400 \\
480 \\
199440 \\
7200 \\
56064 \\
2880 \\
55680 \\
6400 \\
8640 \\
8010 \\
80 \\
4320 \\
1 \\
22080 \\
3461880 \\
465600 \\
17200 \\
4183872 \\
22080 \\
365065 \\
42240 \\
400\end{array}$ \\
\hline
\end{tabular}




\section{Список литературы}

1. Логачев О.А., Сальников А.А., Смышляев С.В., Ященко В.В., Булевы функиии в теории кодирования и криптологии», 2-е изд., М.: МЦНМО, 2012, 584 с.

2. Алексеев Е.К., “О некоторых мерах нелинейности булевых функций”, Прикладная дискретная математика, 2 (2011), 5-16.

3. Алексеев Е.K., "О некоторых алгебраических и комбинаторных свойствах корреляционно-иммунных булевых функций”, Дискретная математика, 22 (2010), 110-126.

4. Алексеев Е.К., Аппроксимачия дискретных функиий алгебраически вырожденными функииями в анализе систем защиты информации, дисс. канд. физ.-матем. наук, 2011.

5. Meier W., Staffelbach O., "Nonlinearity criteria for cryptographic functions", EUROCRYPT'89, 1989, 549-562.

6. Siegenthaler T., "Decrypting a class of stream cipher using ciphertext only", IEEE Trans. Computers, C-34:1 (1985), 81-85.

7. Siegenthaler T., "Correlation-immunity of nonlinear combining functions for cryptographic applications", IEEE Trans. Inf. Theory, IT-30 (1984), 776-780.

8. Шестаков В.И. (ред. перевода), Синтез электронных вычислительных и управляющих систем, М.: ИЛ, 1954.

9. Braeken A., Borissov Y., Nikova S., Preneel B., "Classification of boolean functions of 6 variables or less with respect to cryptographic properties»", Cryptology ePrint Archive, Report 2004/248.

10. Brier E., Langevin P., "Classification of boolean cubic forms of nine variables", IEEE Inf. Theory Workshop (ITW 2003), 2002, 179-182.

11. Maiorana J., "A classification of the cosets of the Reed-Muller code R(1,6)", Math. Comput., 57:195 (1991), 403-414.

12. Harrison M.A., "On the classification of boolean functions by the general linear and affine group", J. SIAM, 12 (1964), 284-299. 